\title{
Occurrence of the pine wood nematode, Bursaphelenchus xylophilus in Portugal and disease spread in Europe.
}

In 1999, the pinewood nematode (PWN), Bursaphelenchus xylophilus was found and identified for the first time in Portugal and in Europe. Following detection, Portuguese authorities initiated the implementation of eradication measures during 1999 and 2000, following an alert provided to European Community officials; as a result, the nematode was confirmed to be confined in the Setúbal region, near Lisbon. A task force from the follow-up group (GANP) created by the Secretary of State for the Rural Development established a national eradication programme (PROLUNP) to (1) Contain PWN within the initial geographic limits; (2) Implement eradication measures; and (3) Monitor PWN at a national level. Research is presently being conducted both at universities as well as research institutes, focusing on the characterization ofBursaphelenchus species associated with maritime pine, as well as on the insect vector, Monochamus galloprovincialis. Recent reports indicate that the nematode may be present in Siberia (Russia), which would present a threat to Eastern European forestry. Efforts are presently being developed by several European countries to establish a research consortium to detect and study the possible presence of PWN, for a new PRAs. A recent workshop held in Portugal, in 2001, has been an oportunity for sharing experiences and techniques on detection and control. There is clearly a greater awareness of this issue in Europe. 